\title{
Design of natural rubber precision ditch fertilization machine
}

\author{
Yeqin Wang ${ }^{1, a}$, Yiguo Deng ${ }^{1, b}$, Yuan Zhang ${ }^{1, c}$ and Lijiao Wei ${ }^{1, d}$ \\ ${ }^{1}$ Institute of Agricultural Machinery, Chinese Academy of Tropical Agricultural Sciences, China \\ a 441213852@qq.com, b dyg7810@21cn.com, c zhang198414@126.com, dweilijiao2008@163.com
}

\section{Keywords: Rubber tree Ditching Fertilize}

Abstract. According to the natural rubber deep fertilization ditch depth of $10 \sim 30 \mathrm{~cm}$ between the width of $30 \sim 40 \mathrm{~cm}$ between the requirements, we developed a fine ditch fertilizing machine. This paper introduces its structure and principle in detail. The machine uses a hoof-shaped furrow structure and centrifugal fertilizer structure. The machine is simple and easy to maintain. After testing, the machine can open the groove width of $20 \mathrm{~cm}$ deep $35 \mathrm{~cm}$ width.The machine fertilizer uniformity up to $90 \%$. The machine productivity reached $1.3 \mathrm{hm}^{2} / \mathrm{h}$. The machine which meets the requirements of rubber tree fertilization has stable performance. At the same time, the machine can be the human's workload.

\section{Introduction}

In China, the natural growth conditions of rubber trees are not as good as in Southeast Asia, such as Malaysia.Rainfall is insufficient.Plot temperature is not high.Soil fertility is relatively poor. The growth period of the rubber tree is relatively short[1,2]. In general, rubber trees need more precision fertilization in China. Rubber soil has low soil nutrient content. Fertilization complements most of the nutrients that rubber trees grow. Farm traditional fertilization habits used for a long time, it is difficult to adapt to changes to the requirements of rubber tree nutrients. Do not fertilize as needed and blindness fertilization, which led to low utilization of fertilizers[3].

In today's world, the plow ditch fertilization machine which structure is bulky and traction resistance is large has been eliminated by some developed countries. As the width and depth of the ditch are limited, the cutter-type ditching machine cannot meet the needs of rubber tree fertilization. Chain 's ditching machine ditch, at the same time, there soil collapse. Vertical ditch fertilizing machine destroys the soil structure is not conducive to the growth of rubber trees[4]. Therefore, in the current management to the field of rubber tree on the fertilization, artificial digging trenches and excavators digging ditches mainly. And we a small amount to the use of hoof ditch plows fertilizer machine. The current rubber tree fertilization is not accurate enough and the structure of the machine more complex has become a major problem for the management of the rubber trees.

Thus, according to the actual needs of the rubber grove ditching and fertilization operations, on the basis of the existing furrow plow, we carried out the research on the precision fertilization machine of rubber tree. The development of a simpler structure, precision fertilization ditch fertilization machine. It can improve production efficiency and reduce production costs.

\section{The overall design of the furrow fertilization machine}

Design Principles. We according to the rubber tree deep fertilization agronomic technical requirements such as ditch depth of $10 \sim 30 \mathrm{~cm}$, width $30 \sim 40 \mathrm{~cm}$ design machine[5]. The machine system structure should be simple and easy to maintain. Its fertilization amount must be controllable and accurate. 
The basic structure of the machine. As shown in Figure 1. The machine consists of four main components: powertrain, trenching system, fertilization system and control system[6]. Powertrain systems include suspension drives and hydraulic drives. The trench is mainly dragged by the tractor to drag the furrow to achieve ditch. Fertilization is the hydraulic drive through the engine power input to the hydraulic motor and hydraulic motor through the worm reducer to power to the fertilizer turntable to achieve fertilization. The control system consists of a hydraulic system and a control circuit system.

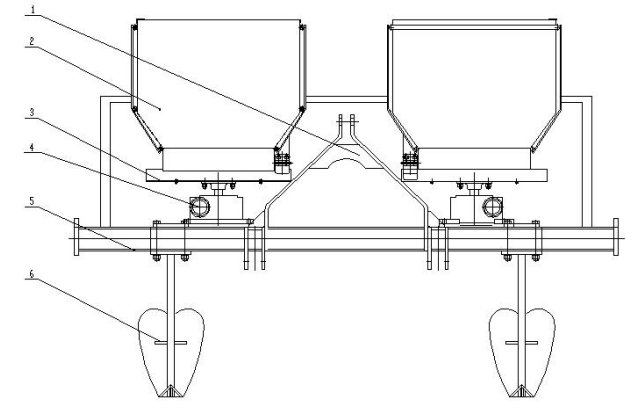

1. Three-point suspension; 2. Fertilizer barrel; 3. Fertilizer turntable; 4. Worm gear reducer; 5. Rack; 6. Ditch furrow

Fig. 1 machine structure diagram

How the machine works. The tractor moves through the three-point suspension mechanism to drag the furrow.In the process of grouting, the soil slabs are cut and formed into v-grooves. Tractor hydraulic output power through the control circuit to adjust the solenoid valve, hydraulic motor rotation, through the chain chain, the drive shaft will be transmitted to the worm gear reducer, worm reducer through the flange, the power transmission to the fertilizer turntable. Rotation process in the fertilizer turntable, fertilizers inside the fertilizer, relying on the role of their own gravity, was directed toward the edge of the fertilizer turntable. The fertilizer on the edge of the fertilizer wheel is scraped to the row of fat. And then through the fat pipe discharged to the disc ditch plow behind the v-shaped groove.

The overall parameters of the machine. The overall parameters of the precision fertilization machine are shown in Table 1.

Table 1 overall technical parameters of the machine

\begin{tabular}{cc}
\hline project & Technical Parameters \\
\hline Dimensions (lengthXwidth X height) $)$ & 1300 X2000X1700 $(\mathrm{mm})$ \\
Supporting power & $\geq 36 \mathrm{hp}$ \\
Ditch width & $20-40 \mathrm{~cm}$ \\
Ditch depth & $10-40 \mathrm{~cm}$ \\
Fertilizer box diameter & $730 \mathrm{~mm}$ \\
Two ditch spacing & $400 \sim 1550 \mathrm{~mm}$ \\
\hline
\end{tabular}

\section{Design of fertilizer system.}

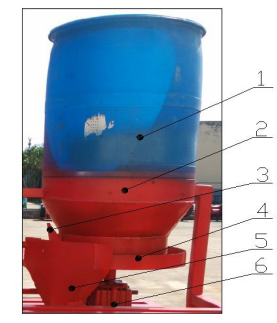

1. Fertilizer bucket; 2. Bucket bracket; 3. Barrier fertilizer; 4. Fertilizer turntable; 5. Row fat bucket 6. Worm gear reducer;

Fig. 2 Structure of Drainage System 
The basic structure and working principle of the fertilizer system. The performance from the fertilizer system directly affects the quality to the work at the fertilizer plant. Comprehensive consideration, uniformity of fertilizer, fertilizer and structural simplification, we choose the fertilizer with the disc rotation, fertilizer baffle-type fertilizer machine[7]. The output of the worm gear reducer is connected with a fertilizer turntable. The top of the fertilizer tray is provided with a barrel holder. The barrel which bottoms into a funnel-type fertilizer bucket, is loaded with a fertilizer bucket on top of the fertilizer tray. The side with the barrel is also connected in a row of fat. The lower end to the row of fat is connected by a fertilizer pipe. There is a fertilizer outlet between the fertilizer bucket and the fertilizer turntable. The deflector baffle is positioned radially along the bucket and is adjustable from the radial spacing of the fertilizer drum. Fertilization system is simple, easy to disassemble, low cost, safe and convenient operation and accurate fertilization. And the fertilizer's bucket can hold a lot of fertilizer. Fertilizer inside the fertilizer depends on its own gravity and fertilizer funnels shape of the role, was directed toward the edge of the fertilizer turntable. Fertilizer on the edge of the turntable fertilizer was blown off the row of fat to the row of fat, and then discharged through the fertilizer pipe.

Fertilizer control system. As shown in Figure 3. The remote control port of the pilot relief valve 1 is connected with a pressure regulating valve 2 and a pressure regulating valve 3 having different set pressures respectively through a three-position four-way switching valve 4. S1, S2, S3 for the control button; K1, K2 for the intermediate relay; 1Y1, 1Y2 for the electromagnetic coil.

Tractor hydraulic system work, the valve in the middle, as shown in the state. The pilot valve 1 is used to set the maximum pressure in the system. The hydraulic motor rotates and the turntable has the fastest speed. K1 for the intermediate relay work, When pressing the button for S1. 1Y2 solenoid coil was energized, the valve at the left position. The pressure is set by the valve 2; the hydraulic motor rotates; the turntable speed is the second. K2 for the intermediate relay work, When pressing the button for S2. 1Y1 solenoid coil was energized, the valve at the right position. The pressure is set by the valve 3; the hydraulic motor rotates; the turntable speed is the slowest[8].
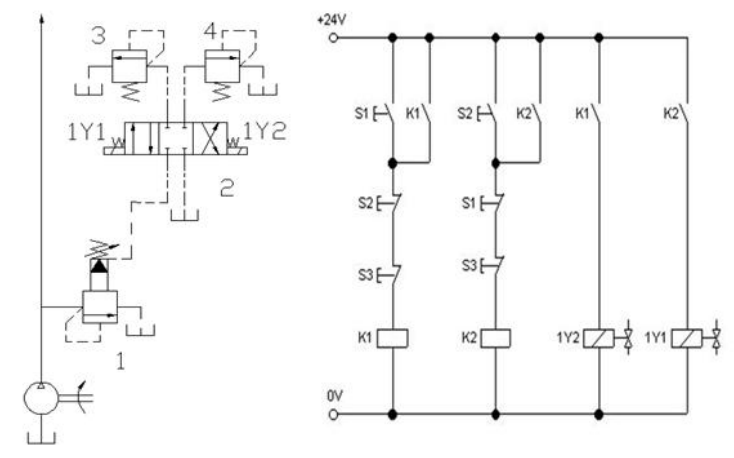

Fig. 3 Technical model of hydraulic electrical control for fertilizer system

Characteristics of Drainage Control System. To use of electrical control, the system response is fast and action is accurate. Use three pilot relief valves for remote connection, so that the system has three different pressure set value, its set the turntable speed. According to the different characteristics of the different speed rows of fertilizer, you can adjust the turntable speed to determine the amount of fertilizer to achieve precise control of fertilizer.

Fertilizer test. Experimental site in Xuwen County,Zhanjiang City, Guangdong Province, the Friendship Farm flat cement land. We use the equipment is the East Red 604 tractor. We use fertilizers for powdered phosphate fertilizer, potash and granular compound fertilizer. We measured the machine under different operating speed of fertilizer, fertilizer uniformity. The machine travels 
across $600 \mathrm{~m}$ at normal operating speed $(0.7 \mathrm{~m} / \mathrm{s}, 0.9 \mathrm{~m} / \mathrm{s}, 1.1 \mathrm{~m} / \mathrm{s})$, takes 90 points, and the length of each point is $1 \mathrm{~m}$.

Table 2 Machine Fertilization Test Summary Table

\begin{tabular}{ccc}
\hline Advance speed $(\mathrm{m} / \mathrm{s})$ & Turntable speed (r/min) & $\begin{array}{c}\text { Row of } \\
\text { fertilizer }(\mathrm{g} / \mathrm{m})\end{array}$ \\
\hline 0.7 & 6 & 120 \\
0.7 & 9 & 228 \\
0.7 & 14 & 256 \\
0.9 & 6 & 69 \\
0.9 & 9 & 154 \\
0.9 & 14 & 169 \\
1.1 & 6 & 35 \\
1.1 & 9 & 85 \\
1.1 & 14 & 97 \\
\hline
\end{tabular}

Fertilizers are centrifuged on the fertilizer turntable.

$$
F=m \frac{w^{2}}{r}=\frac{4 m \pi^{2} n^{2}}{r}=a m
$$

$\mathrm{F}$ is the centripetal force of the fertilizer particles; $\mathrm{m}$ is the mass of the fertilizer particles; $\mathrm{w}$ is the turntable angular velocity; $r$ is the turntable radius; $n$ is the turntable speed; $a$ is the fertilizer particle acceleration; From the formula (1) can be obtained turntable speed of the impact of fertilizer.

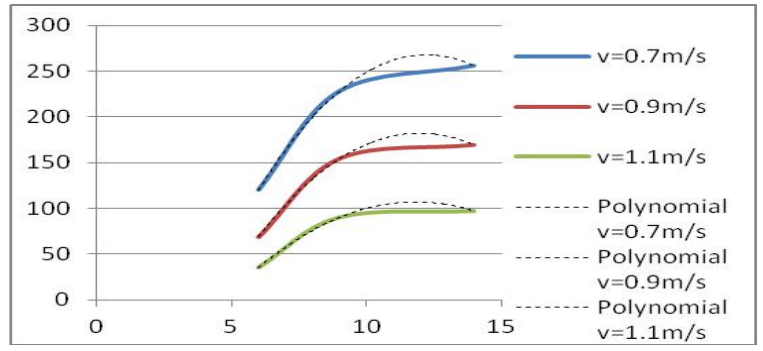

Fig. 4 Effect of turntable speed on fertilizer

We used SAS (statistical analysis system) statistical analysis software to test the data in Table 2 polynomial regression analysis; and draw the response surface map. We can observe the impact of various factors on the amount of fertilizer, and be able to determine and test the relationship between the variables[9].

We according to Figure 4 turntable speed on the impact of fertilizer and Table 2 machine fertilization test summary of the data in a single-factor analysis. The polynomial regression equation is as follows: (2) (3) (4)

When the tractor at the forward speed $\mathrm{v}=0.7 \mathrm{~m} / \mathrm{s}$, the row of fertilizer $\mathrm{y}$ and turntable speed $\mathrm{x}$ relationship as shown in formula (2):

$$
y=-26.6 x^{2}+651 x-2108.4
$$

When the tractor at the forward speed $\mathrm{v}=0.9 \mathrm{~m} / \mathrm{s}$, the row of fertilizer $\mathrm{y}$ and turntable speed $\mathrm{x}$ relationship as shown in formula (3):

$$
y=-28.5 x^{2}+682.5 x-2448
$$

When the tractor at the forward speed $\mathrm{v}=1.1 \mathrm{~m} / \mathrm{s}$, the row of fertilizer $\mathrm{y}$ and turntable speed $\mathrm{x}$ relationship as shown in formula (4):

$$
y=-20.35 x^{2}+492.25 x-1835.9
$$


According to the polynomial regression equation (2) (3) (4), it is found that the amount of fertilizer increases as the increase of the rotational speed of the turntable, and reaches a certain value and decreases with the increase of the rotational speed of the turntable.

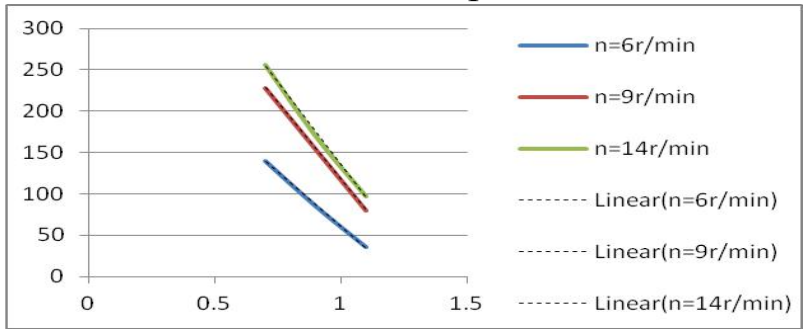

Fig. 5 The Influence of the Tractor 's Forward Speed on the Fertilizer

According to the data of Fig. 5 and Table 2, the single-factor analysis was carried out. When the turntable speed is fixed, the amount of fertilizer is proportional to the speed of the tractor, and the amount of fertilizer decreases as the tractor advances.

\section{Field trials}

Basic conditions of field trials. The test site is in the rubber farm of the Friendship Farm. We use the equipment is the East Red 604 tractor. We use fertilizers for powdered phosphate fertilizer, potash and granular compound fertilizer. We measured the performance on the machine, fertilizer uniform performance and precision fertilization situation[10].
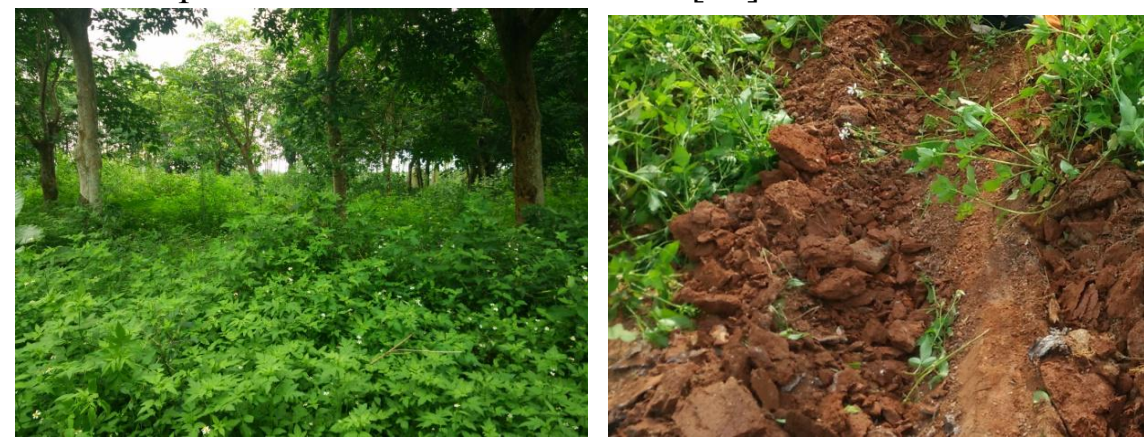

Fig. 6 Drainage test before and after the map

Field test is divided into two parts: First, in the case of no fertilization, different forward speed, ditch effect test; Second, both open ditch and fertilization case, test ditch fertilization comprehensive effect.

\section{Field test results}

\begin{tabular}{cc} 
Table 3 Field test results summary table \\
\cline { 2 - 2 } project & test results \\
\hline Supporting power & $60 \mathrm{hp}$ \\
Ditch width & $35 \mathrm{~cm}$ \\
Ditch depth & $20 \mathrm{~cm}$ \\
Fertilizer & $\geq 90 \%$ \\
uniformity & \\
V $=0.9 \mathrm{~m} / \mathrm{s}$ & $151 \sim 158 \mathrm{~g} / \mathrm{m}$ \\
fertilizer & \\
Two ditch spacing & $1050 \mathrm{~mm}$ \\
productivity & $1.3 \mathrm{hm}^{2} / \mathrm{h}$ \\
\hline
\end{tabular}

The experimental analysis shows that the machine meets the rubber glue garden ditch fertilization operation. Machine fertilizer is more uniform. Plastic workers can refer to Table 2 machine fertilization test summary table to control the tractor forward and turntable speed kneading 
garden precision fertilization. The productivity reached $1.3 \mathrm{hm} 2 / \mathrm{h}$, which was obviously higher than that of digging and digging.

\section{Conclusions}

According to the agronomic requirements of rubber fertilization, we designed a grooving to plow furrow and turntable fertilizer of rubber tree ditch fertilization machine. Through the field test, the machine to meet the rubber deep fertilization ditch depth of $10 \sim 30 \mathrm{~cm}$ between the width of $30 \sim$ $40 \mathrm{~cm}$ between the requirements. At the same time, we can refer to the test data to precision fertilization. The machine has reached the desired design requirements. In the next research and development process, we mainly on the fertilizer monitoring and discharge action of the feedback control to study.

\section{Acknowledgements}

This work was financially supported by the Natural Science Foundation of Hainan Province(No.20165204),Science and Technology Project of Zhanjiang City(No.2015A03023), the public welfare research and capacity-building special funds for Guangdong Province(No.2017A020208012),Central Public-interest Scientific Institution Basal Research Fund for Chinese Academy of Tropical Agricultural Sciences ( No.1630022015023\&1630132016015\&1630132017003) and Collaborative innovation and platform environment construction special of Guangdong Province (No.2017A040406003).

I would like to thank the members of the research group for their selfless help, especially the communication author Yiguo Deng, associate professor and the Friendship Farm in Guangdong Province to give the strong support.

\section{References}

[1] Ning Lin, Quanfa Ma, Yunping Lin, et al. Low-yield rubber plant fertilization transformation experiment [J]- China Tropical Agriculture 2009 (2)

[2] Min Wu. Peng He. Jiabao Wei . Comprehensive Evaluation of Soil Fertility in Hainan Island [J] - China Soil and Fertilizer 2009 (2)

[3] Pengyi Li. Zhi Huang. Haijian Chen. Liqian Wang. Application and Discussion on Fertilization Model of Modern Rubber Garden [J] China Tropical Agriculture 2013

[4] Chaohai Chen, Jieli Duan, Guoqi Yan, et al. The present situation and development strategy of orchard ditching [J] modern agricultural equipment, 2013 (2)

[5] LiuFang Shentu, Gang Yang, Xingzhao Sun, vineyard special ditch fertilization machine design [J], agricultural mechanization research 2012 (11)

[6] Limin Yan, Yang Liu, Yaxiong Li, et al. Design and experiment of grape furrow fertilizing machine [J], Anhui Agricultural Sciences, Journal OfAnhuiAgri. Sci. 2015, 43 (3)

[7] Zhaomin Qin, Junhui Liu. Research and Design of Sprinkler Parts of Centrifugal Sprinkler [J] agricultural mechanization research 2006(10)

[8] Yimin $\mathrm{Xu}$, hydraulic motor balance circuit braking characteristics analysis [J], machine tools and hydraulic, 2006 (8): 161-162

[9] Qing-xiang LUAN, Yang ZHAO, Xin ZHOU, et al.Evaluation of the best extraction process of Eucommia ulmoides by single factor test combined with response surface analysis [J]. Chinese Journal of Pharmaceutical Analysis, 2013,33 (5): 859-865.

[10] Ming Li, Ting Zhang, Xuehu Dong, et al. Optimization of the parameters of 3ZSP - 2 type sugarcane cultivated fertilization applicator scraper type fertilizer[J]. Journal of Agricultural Engineering, 2016,32 (23): 36-42 